\title{
The role of polyunsaturated lipids in membrane raft function
}

\author{
William Stillwell \\ Department of Biology, Indiana University-Purdue University, Indianapolis, USA
}

\begin{abstract}
Docosahexaenoic acid (DHA), with 22 carbons and six double bonds, is the longest, most unsaturated fatty acid commonly found in humans. It represents the extreme example of an omega-3 (n-3) polyunsaturated fatty acid. Since early epidemiology studies, DHA has been linked to alleviation of an enormous number of human afflictions, including heart disease, cancer and neurological disorders. How one simple molecule can affect so many seemingly unrelated abnormalities has been a contentious question for many years. One research direction has investigated events that follow the uptake of DHA into animal cell plasma membrane phospholipids. Summarized here is a variety of membrane properties impacted by the incorporation of DHA. DHA's dynamic shape, consisting of multiple configurations, is very different from what its static, stick structure would indicate. DHA-containing phospholipids have a wide hydrophobic base compared with their hydrophilic head and so induce negative curvature strain that severely impacts the activity of a variety of important membrane proteins. The unusual structure means that DHA-rich membranes are also surprisingly thin and support high permeability, compression, fusion and flip-flop rates. DHA does not exist in an environment that is independent from other membrane lipids. Of particular interest is the interaction of DHA-containing phospholipids with the major lipid raft components cholesterol and sphingomyelin. From a wide variety of biophysical studies, primarily done on model lipid monolayers and bilayers, a new hypothesis is proposed suggesting that DHA may alter plasma membrane lipid raft structure and hence essential cell signaling events. A fundamental role for DHA affecting a feature common to all cells, membrane structure and function, may explain its wide variety of reputed health benefits.
\end{abstract}

Keywords: docosahexaenoic acid; lipid rafts; membrane structure; polyunsaturated fatty acids; $n$ - 3

Abbreviations: AFM: atomic force microscopy; DHA: docosahexaenoic acid; DRM: detergent-resistant membrane; DSM: detergent-soluble membrane; OA: oleic acid; PC: phosphatidylcholine; PE: phosphatidylethanolamine; PP: protein phosphatase; PS: phosphatidylserine; PUFA: polyunsaturated fatty acid; SM: sphingomyelin.

\section{Introduction}

$\mathbf{P}$ olyunsaturated fatty acids (PUFAs) play an essential role in maintaining human health. Of particular interest are the family of long-chain PUFAs that have their last double bond three carbons from the methylene or omega end. These fatty acids, regardless of chain length or total number of double bonds, are known as omega-3 (n-3) fatty acids, and are believed to play special but still undefined roles in human health. For almost 20 years the author's laboratory has studied the longest and most unsaturated of the n-3 PUFAs, docosahexaenoic acid (DHA). This article will briefly summarize one possible mechanism of action for DHA related to these studies.

\section{Health benefits and distribution of docosahexaenoic acid}

With 22 carbons and six double bonds, DHA $\left(22: 6^{\Delta 4,7,10,13,16,19}\right)$ is the longest and most unsaturated fatty acid commonly found in biological systems (1). Since DHA represents the extreme version of an n-3 fatty acid, it should be easier to deduce its mode of action than for the other, shorter and less unsaturated n-3 fatty acids. DHA's first association with human health was in the classic epidemiological studies of Bang et al. (2) on Eskimos. The early studies linked diminished heart disease and cancer to consumption of enormous quantities of coldwater fish oil found in the Eskimo diet. Since then fish oils and particularly DHA have 
Table I. Human afflictions associated with low docosahexaenoic acid levels (3)

\begin{tabular}{|c|c|c|}
\hline Aggression & Dermatitis & Phenylketonuria \\
\hline Alcoholism & Dyslexia & $\begin{array}{l}\text { Problems with brain } \\
\text { development }\end{array}$ \\
\hline Arthritis & Gingivitis & $\begin{array}{l}\text { Problems with neurovisual } \\
\text { development }\end{array}$ \\
\hline Asthma & Heart disease & Psoriasis \\
\hline $\begin{array}{l}\text { Attention deficit } \\
\text { hyperactivity disorder }\end{array}$ & Hypersensitivity & Reduced fertility \\
\hline Bipolar disorder & Kidney disease & Respiratory diseases \\
\hline Blindness & Lupus & Schizophrenia \\
\hline Cancer & Malaria & Suicide \\
\hline Crohn's disease & Migraine headaches & Ulcerative colitis \\
\hline Cystic fibrosis & Multiple sclerosis & Zellweger's syndrome \\
\hline Depression & Nephropathy & \\
\hline
\end{tabular}

been linked to alleviation of a vast array of human afflictions (3), some of which are listed in Table 1. The question that immediately arises is how one simple molecule can affect so many seemingly unrelated pathologies. To accomplish this DHA must be affecting basic processes common to many cell types. Proposed modes of action for DHA can be roughly divided into five, non-exclusive categories (4). DHA has been suggested to affect eicosanoid biosynthesis (5), protein activity through direct interaction (6), protein activity indirectly through altering transcription events (7), lipid peroxidation products (8), and membrane structure and function (4). Here, the discussion will be limited to the effect of DHA on membrane structure and function.

At first glance one might predict that at a very long 22 carbons, membranes rich in DHA would be exceptionally thick. Surprisingly, DHA-rich membranes such as the rod outer segment are actually quite thin (9). This is due to DHA's unusual threedimensional structure. In Fig. 1 the structure of DHA is drawn in several ways. Fig. 1(A) presents the conventional stretched stick structure. A more realistic bent stick structure depicting bending at the six cis double bonds is shown in Fig. 1(B), while a molecular dynamics study (Fig. 1C) depicts two of the many configurations associated with DHA (10). Several of DHA's possible configurations even have the omega end bent up to the aqueous interface. As a result, the actual structure of DHA is a pyramid with a wider base than head (Fig. 1D). This structure not only produces a thin membrane but also explains DHA's preference for non-lamellar
Docosahexaenoic Acid Structure
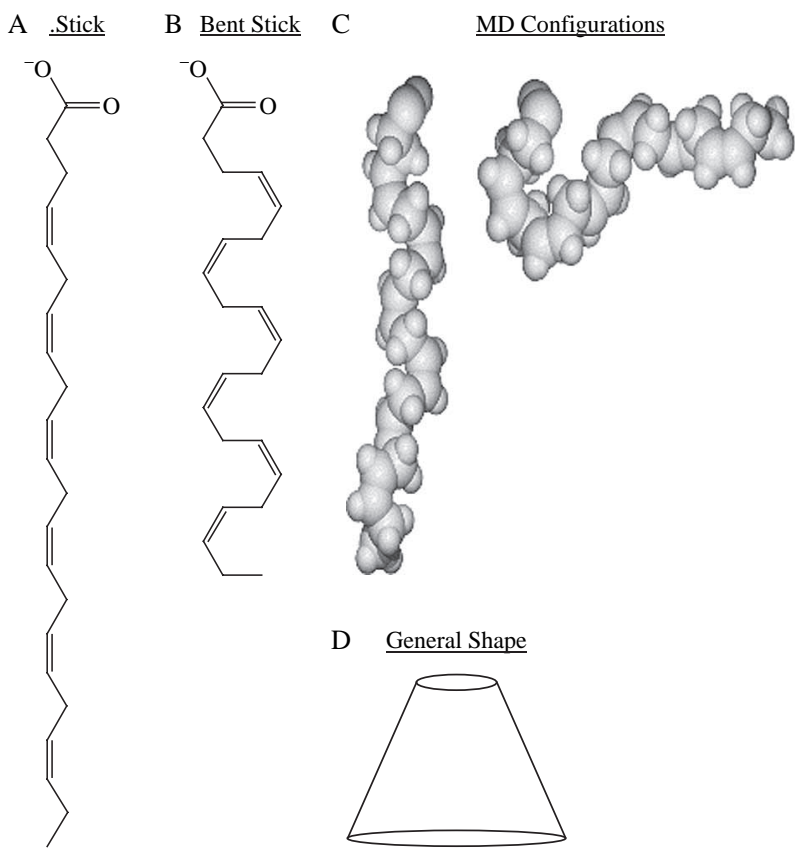

Fig. 1. Docosahexaenoic acid structures: (A) conventional stretched stick; (B) bent stick; (C) two conformations from molecular dynamics (MD); (D) cartoon pyramid. (The author thanks Dr S Feller, Wabash College, for the molecular dynamics figures depicted in $1 \mathrm{C}$.)

$\left(\mathrm{H}_{\mathrm{II}}\right)$ phase (11) and its ability to induce negative curvature stress in adjacent proteins (12).

As is typical of all biological compounds, DHA is not homogeneously distributed throughout the whole body, organs, tissues, cells or cell membranes. Extremely high levels of DHA, occasionally approaching half of the total acyl chains present, are found in the sperm, rod outer segment and neuronal synaptosomes (1). DHA levels are so high in these cells that the plasma membrane phospholipids often have DHA located in both acyl chains (13). This motif is unusual, as in most biological phospholipids the $s n-1$ chain is saturated while the $s n-2$ chain is unsaturated (14). Also in these high-DHA tissues, the DHA level is relatively insensitive to dietary manipulation and once incorporated the high level is tenaciously retained at the expense of other fatty acids. In sharp contrast are the other body tissues and cells that have much lower inherent levels of DHA, usually less than a few per cent of the acyl chains. Here, DHA occupies primarily the $s n-2$ position and few di-DHA phospholipids exist. These tissues are highly sensitive to the diet, and so it is here that the reasons behind DHA's health effects may be found. 
It is well documented that DHA can be readily incorporated into the phospholipids of many cell membranes, particularly the plasma and mitochondrial membranes (4). Once again, DHA's incorporation is not homogeneous and varies depending on the cell and membrane type as well as the phospholipid class. In most reported experiments DHA is primarily esterified to phosphatidylethanolamine (PE) and phosphatidylcholine (PC), with incorporation also reported into the other, less abundant phospholipids $(3,4,15)$. However, this is not a hard rule as DHA has been reported initially to incorporate into phosphatidylserine (PS) in synaptosomes (1). The preference of DHA for the primary amine phospholipids PE and PS is of particular interest since these lipids are primarily associated with the plasma membrane inner leaflet (16). It is likely that DHA exhibits a transmembrane heterogeneity favoring the inner leaflet, while the lipid rafts, discussed below, are found in the outer leaflet.

\section{Effect of docosahexaenoic acid on membrane physical properties}

All fatty acids are known to be able to affect cells through the "detergent effect". Therefore, if cultured cells are incubated with sufficiently high quantities of free fatty acids, they die. The fatty acid level necessary to induce cytotoxicity directly varies with fatty acid and cell type and the level of serum albumin in the culture media. Of all fatty acids, DHA appears to be particularly cytotoxic (17). Much lower levels of DHA exposed to the cells for longer periods can also kill cells eventually through the initiation of apoptotic pathways (3) (discussed below).

So how does DHA function at the molecular level? Over the past 30 years or so, the direct effect of DHA on membrane structure has been investigated from many angles, using a variety of complex biophysical techniques in many laboratories throughout the world (4). The purpose of this review is not to discuss how any of these often esoteric techniques work, but rather to give the reader a general feel of how DHA affects some important membrane properties. Table 2 compares the effect of DHA with the most abundant unsaturated membrane fatty acid, oleic acid (OA, $\left.18: 1^{\Delta 9}\right)$. Comparisons are made on a few basic membrane properties including cross-sectional area, acyl chain length, permeability, fusion, compressibility and flip-flop (3). Although OA and DHA
Table 2. Effect of docosahexaenoic acid (DHA) on some basic membrane physical properties (3)

\begin{tabular}{ll}
\hline & DHA/OA \\
\hline Cross-sectional area & 1.13 \\
Chain length & 0.95 \\
Permeability (water) & 2.66 \\
Fusion & 8.55 \\
Compressibility $^{\mathrm{a}}$ & 0.55 \\
Flip-flop & 39.3 \\
\hline
\end{tabular}

a Compressibility reflects the energy required to compress the fatty acyl chain. DHA requires less energy than oleic acid $(O A)$ and so its DHA/OA ratio is less than 1.0 .

both affect membrane structure, DHA is much more effective. Owing to DHA's multiple configurations and pyramid shape, DHA-rich membranes are thin and fluid, have looser lipid packing, may prefer non-lamellar $\left(\mathrm{H}_{\mathrm{II}}\right)$ phases, induce negative curvature strain to proteins, and in general are more dynamic. Increased water permeability, for example, makes DHA-rich membranes "wetter" (18). The high fusion rates reported for DHA-rich model membranes are consistent with the fatty acid's location in membranes known to exhibit naturally high levels of fusion or the reverse process of exfoliation (sperm, rod outer segment and synaptosomes).

\section{Interaction of docosahexaenoic acid with cholesterol/lipid rafts}

A biological membrane consists of many hundred different lipid species and a hundred or so proteins, heterogeneously distributed, and all components are in constant flux. As a result, upon its incorporation into membranes, DHA will be exposed to many different lipids and proteins. Its interactions with other membrane components, favorable and unfavorable, will affect membrane heterogeneity (domain structure) and hence, it is believed, cell function.

The author's group initially concentrated on the interaction of DHA-containing PCs and PEs with cholesterol (19). Cholesterol was chosen owing to its integral role in maintaining the structure and function of plasma membrane lipid rafts (20). At present, lipid rafts form the major basis for discussing membrane structure. Unfortunately, there is no consensus of what a raft actually is (21). Cell biology and membrane biophysics approaches are often contradictory. More than three 
decades ago the famous Cornell biochemist Efraim Racker had this to say about another poorly understood process during this time, oxidative phosphorylation (22): "Anyone who is not confused about oxidative phosphorylation just does not understand the situation." By substituting "oxidative phosphorylation" with "lipid rafts", the current state of affairs on membrane structure is defined. Lipid rafts are a type of plasma membrane lateral heterogeneity (domain) that is involved in cell signaling (20). Rafts are enriched in cholesterol, the glue that holds them together, sphingolipids and saturated chain phospholipids, and so are found in a highly structured liquid-ordered $\left(1_{\mathrm{o}}\right)$ state. Characteristic families of signaling proteins are found associated with rafts and are a standard by which rafts are categorized. Rafts are operationally defined by their insolubility in cold non-ionic detergents, primarily Triton X-100. Therefore, rafts are also referred to as detergent-resistant membranes (DRMs). The presence of cholesterol is also a universal trait of rafts, as its removal by cyclodextrin marks raft demolition. Since DHA chains disrupt lipid packing, membrane bilayers containing this fatty acid exist in the liquid-disordered $\left(l_{d}\right)$ state. The author's studies are based on the assumption that DHA-containing phospholipids should phase separate away from cholesterol-rich rafts and so should form domains that are very different from $l_{\mathrm{o}}$ state rafts. These domains would be soluble in cold detergent [detergent-soluble membranes (DSMs)] and would have physical properties and resident proteins considerably different from those of rafts. As the DHA level would rise with dietary consumption of fish oil, the organization of plasma membranes into raft and non-raft domains would change, thus altering the cells' physiology.

Many types of experiment have demonstrated DHA's aversion for cholesterol. Here, recent X-ray studies done as a collaboration between the author's research group in Indianapolis and Martin Caffrey's at the Ohio State University will be described (3), followed by a discussion of some DRM and DSM studies (23) and a preliminary atomic force microscopy (AFM) study that confirm these results (23).

To determine the membrane-carrying capacity of cholesterol, liposomes were made from DHA-containing PCs and PEs to which increasing molar percentages of cholesterol were added. At some critical molar percentage, the membrane solubility of cholesterol is exceeded and any additional cholesterol appears outside the membrane as cholesterol monohydrate crystals, which can be accurately detected by X-ray diffraction (3). For the heteroacid PCs the cholesterol solubility limit is $65 \mathrm{~mol} \%$ in $16: 0,18: 1 \mathrm{PC}$, but only $55 \mathrm{~mol} \%$ in $16: 0$, 22:6 PC. In PE bilayers cholesterol solubility is lower than in PCs, being $\sim 51 \mathrm{~mol} \%$ on $16: 0,18: 1$ $\mathrm{PE}$, and is substantially reduced to $31 \mathrm{~mol} \%$ in $16: 0$, 22:6 PE bilayers. If DHA occupies both chains, cholesterol membrane solubility is further decreased to $11 \mathrm{~mol} \%$ for $22: 6,22: 6 \mathrm{PC}$ and $8.5 \mathrm{~mol} \%$ for $22: 6$, 22:6 PE. These X-ray diffraction studies indicate that DHA is not compatible with cholesterol. Supporting this conclusion are cyclodextrin extraction studies from Niu et al. (24). These investigators reported that cholesterol can be extracted from bilayers composed of 16:0, 22:6 PC about 1.9 times more easily than from those made of 16:0, 18:1 PC.

Cold-temperature, non-ionic detergent extraction is historically the hallmark of lipid rafts (21). To test the partitioning of DHA between raft and non-raft fractions, model plasma membranes were made of either 16:0, 18:1 PE/sphingomyelin (SM)/cholesterol or 16:0, 22:6 PE/SM/cholesterol (23). Fig. 2 presents data from cold-temperature Triton X-100 extractions performed on the two model plasma membranes. Consistent with the raft hypothesis, very little $(<10 \%)$ of the SM or cholesterol is extracted into the detergent-soluble (DSM), non-raft fraction for either model membrane. Instead, both SM and

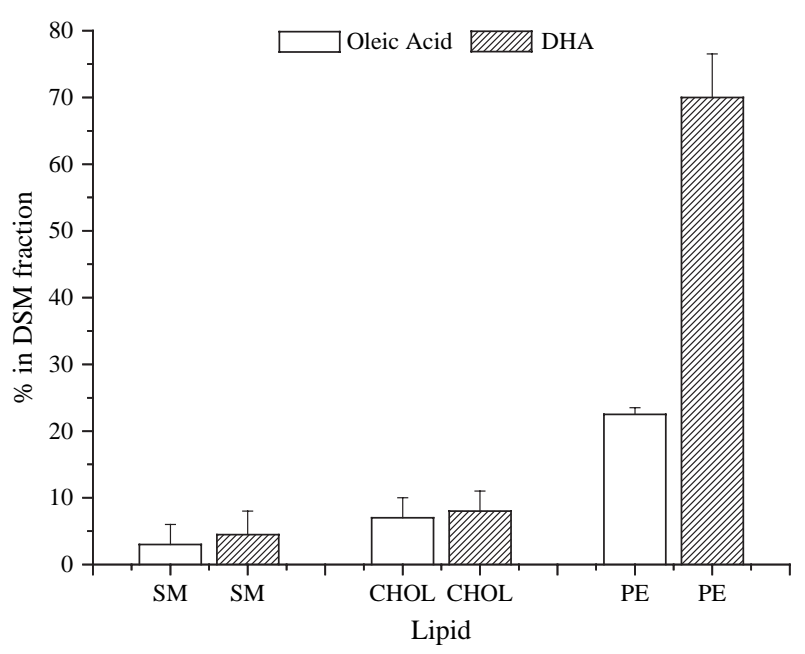

Fig. 2. Membrane fractions soluble in cold $\left(4^{\circ} \mathrm{C}\right)$ Triton $\mathrm{X}-100$ [detergent soluble membrane (DSM) fractions]. Membranes were composed of $1: 1: 1(\mathrm{~mol} / \mathrm{mol} / \mathrm{mol})$ of sphingomyelin $(\mathrm{SM}) / \mathrm{cholesterol}$ (chol)/phosphatidylethanolamine (PE); the clear bars are from 16:0, 18:1 PE-containing membranes and the hatched bars are from 16:0, 22:6 PE-containing membranes. DHA: docosahexaenoic acid. 
cholesterol are found in the detergent-resistant (DRM), raft fraction. There is a very noticeable difference in partitioning of the OA- and DHA-PEs. Approximately $70 \%$ of the $16: 0,22: 6 \mathrm{PE}$ is associated with the DSM (non-raft) fraction, while 68\% of the 16:0, 18:1 PE locates into the DRM (raft) fraction. This implies that there is greater phase separation from rafts of DHA-PE than of OA-PE.

AFM studies were initiated to visualize directly any possible DHA-related membrane domains (24). the model plasma membranes again consisted of either 16:0, 18:1 PE/SM/cholesterol (1:1:1) or 16:0, 22:6 PE/SM/cholesterol (1:1:1). Domains are clearly visible (Fig. 3). The SM/cholesterol lipid rafts are thicker (by $\sim 1.1 \mathrm{~nm}$ ) than the surrounding non-raft regions and appear lighter in color. A rough estimate places the raft area for the 16:0, 18:1 PE/ $\mathrm{SM} /$ cholesterol $(1: 1: 1)$ membrane at $\sim 26 \%$ compared with $\sim 59 \%$ for the $16: 0,22: 6 \mathrm{PE} / \mathrm{SM} /$ cholesterol $(1: 1: 1)$ membrane. The conclusion is that DHA makes the raft domains larger and they are interconnected.

Finally, these DHA-raft studies have very recently been extended to another membrane lipid component, $\alpha$-tocopherol (vitamin E) (author's unpublished observation). $\alpha$-Tocopherol is the traditional membrane lipid antioxidant. Since DHA has six double bonds, it is highly susceptible to oxidation and must be protected by antioxidants. Because DHA partitions into non-raft domains, one would predict that $\alpha$-tocopherol should track with DHA. It would make little biological sense if $\alpha$-tocopherol partitioned into highly saturated rafts. Differential scanning calorimetry was used to test this hypothesis by comparing the affinity of $\alpha$-tocopherol and cholesterol for raft (16:0 SM) and non-raft (16:0, 22:6 PC) lipids. Liposomes were made from a $1: 1(\mathrm{~mol} / \mathrm{mol})$ ratio of 16:0 SM and 16:0, 22:6 PC to which increasing amounts of either $\alpha$-tocopherol or cholesterol were added. The two base lipids were chosen because not only do they represent non-raft and raft plasma membrane components, but also their melting points $\left(T_{\mathrm{m}}\right)$ are far enough apart that even in a mixture they can be clearly distinguished (Fig. 4). As cholesterol is added to the $1: 1$ mixture of 16:0 SM and 16:0, 22:6 PC the higher melting SM transition is obliterated, indicating a preferential affinity of cholesterol for SM. In sharp contrast, as $\alpha$-tocopherol is added, the lower melting DHA-containing PC is obliterated,
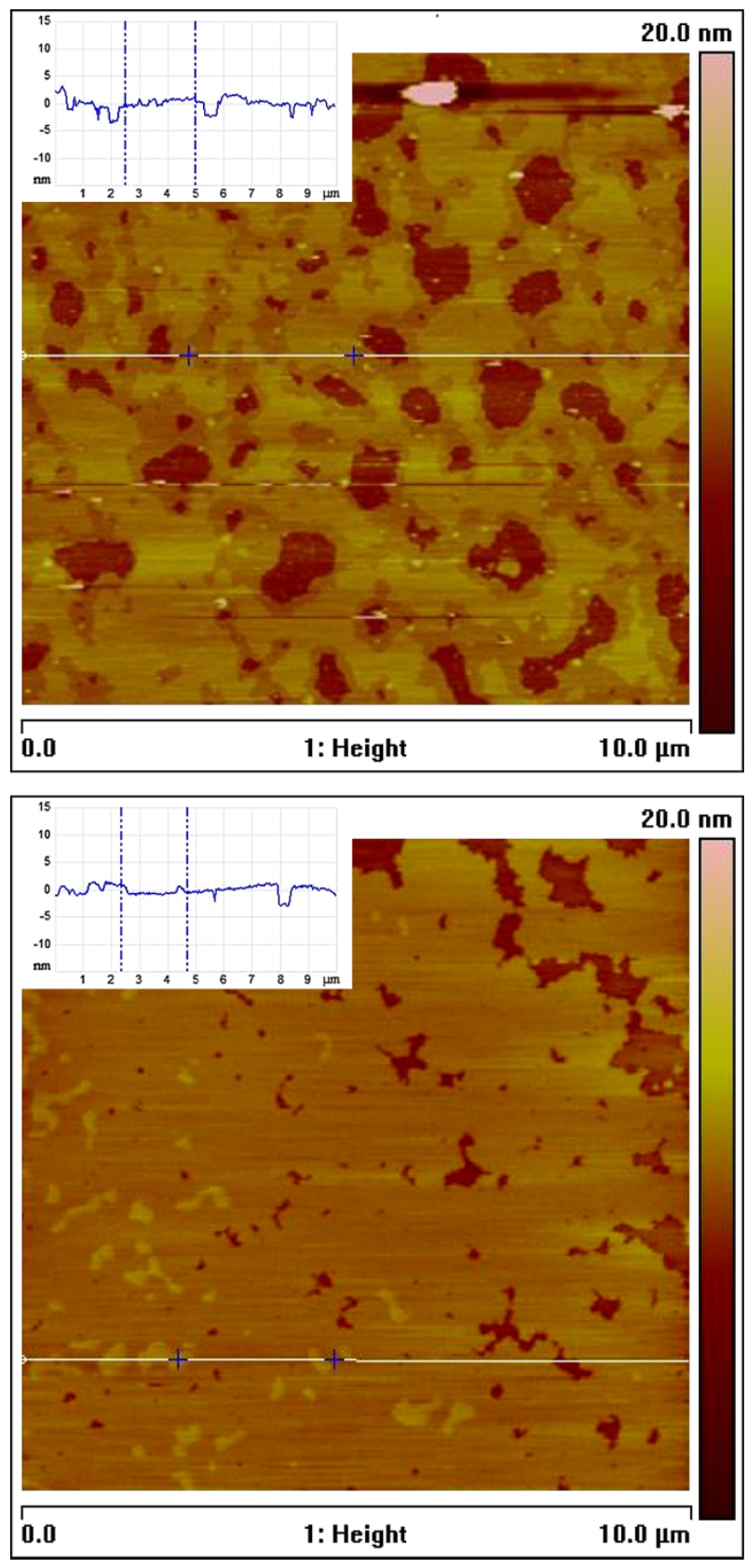

Fig. 3. Atomic force microscopy images of mica-supported bilayers composed of 16:0, 18:1 phosphatidylethanolamine (PE)/sphingomyelin (SM)/cholesterol $(1: 1: 1, \mathrm{~mol} / \mathrm{mol} / \mathrm{mol})$ (top) and 16:0, 22:6

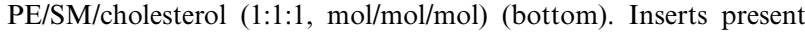
the height profiles corresponding to the horizontal white lines in the images.

indicating a preferential affinity of $\alpha$-tocopherol for DHA. These experiments support the hypothesis that polyunsaturated phospholipids may phase separate from lipid rafts and as a consequence may create $1_{d}$ domains that have a totally different set of components and functions from lipid rafts. 


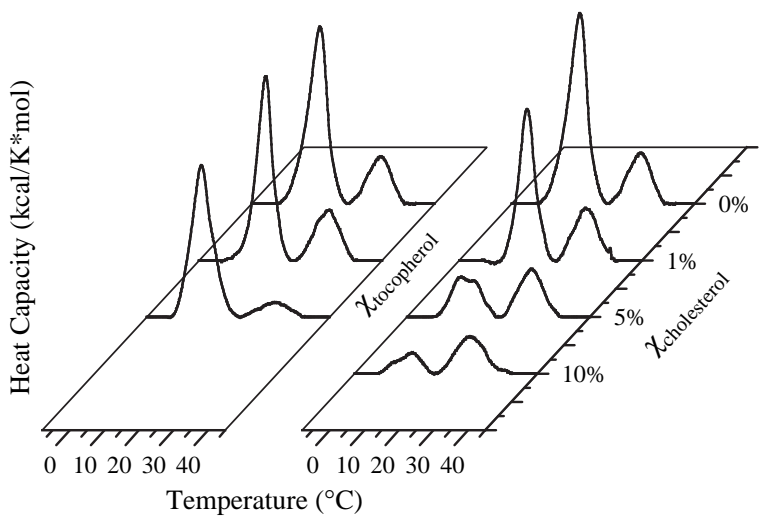

Fig. 4. Comparison of the affinity of $\alpha$-tocopherol and cholesterol for raft [16:0 sphingomyelin (SM)] and non-raft [16:0, 22:6 phosphatidylcholine (PC)] lipids. Membranes were made from a $1: 1(\mathrm{~mol} / \mathrm{mol})$ ratio of $16: 0 \mathrm{SM}$ and 16:0, 22:6 PC to which increasing amounts of either $\alpha$-tocopherol or cholesterol were added. Differential scanning calorimetry cooling curves are depicted. The higher melting transition is due to a 16:0 SM-rich component, while the lower melting transition is due to the 16:0, 22:6 PE-rich component. This experiment indicates that cholesterol has a preferential affinity for SM (raft), while $\alpha$-tocopherol prefers the DHA-PE (non-raft) components.

\section{Effect of docosahexaenoic acid on signaling pathways}

There is an ever increasing number of reports linking DHA to alterations of a series of reactions constituting a pathway. For more than 15 years Mitchell and Litman (25) have investigated the effect of DHA on rhodopsin and the G-proteincoupled visual receptor pathway. Siddiqui and coworkers (reviewed in ref. 3) reported the effect of DHA on signaling pathways involved in apoptosis of cancer cells and hypertrophy in cardiomyocytes. By one pathway in Jurkat leukemic cells, low levels of DHA stimulate the activity of sphingomyelinase, increasing production of ceramide. Increased ceramide leads to inhibition of cdk-2 activity and stimulation of the protein phosphatases PP1 and PP2A, which in turn decreases the net phosphorylation of retinoblastoma protein. Consequently, Jurkat cell growth is arrested. DHA was also reported to enhance apoptosis through a mitochondria-dependent pathway. A DHA-induced increase in ceramide causes activation of pro-apoptosis Bcl-2 proteins, which then leads to release of cytochrome $\mathrm{C}$ from the mitochondria and eventual activation of caspase 3 with downstream execution of the apoptotic caspase cascade. In cardiomyocytes, low levels of DHA prevented phenylephrine-induced hypertrophy by inhibiting the Ras $\rightarrow$ Raf $1 \rightarrow$ Erk1/2 $\rightarrow$ $\mathrm{p} 90^{\mathrm{rsk}} \rightarrow$ hypertrophy pathway. These are but a few examples of DHA-affected signaling pathways and undoubtedly many more examples will be forthcoming in the future.

\section{Summary}

As a normal component of the human diet, DHA can be readily incorporated into phospholipids that comprise biological membranes. DHA's unusual shape (multiple configurations resulting in a net pyramid shape) severely impacts lipid packing and hence membrane physical properties. DHA's strong aversion to cholesterol results in enhanced phase separation, altering the size and composition of rafts as well as forming a unique DHA-rich, highly disordered, non-raft phase. DHA-induced alterations in membrane structure then affect cellular biochemical activity, including essential signaling pathways, accounting for the health benefits of n-3 PUFAs.

\section{Acknowledgements}

The author wishes to acknowledge support from the National Institutes of Health (grant no. CA 57212).

\section{References}

1. Salem N Jr, Kim H, Yergey JA. Docosahexaenoic acid: membrane function and metabolism. In: Simopolous AP, Kifer RR, Martin RE, eds. Health effects of polyunsaturated fatty acids in seafoods. New York: Academic Press; 1986. p. 319-51.

2. Bang HO, Dyerberg J, Hjoorne N. The composition of food consumed by Greenland Eskimos. Acta Med Scand 1976; 200: 69-73.

3. Stillwell W, Shaikh SR, LoCascio D, Siddiqui RA, Seo J, Chapkin RS, Wassall SR. Docosahexaenoic acid: an important membrane-altering omega-3 fatty acid. Hauppage, NY: NOVA Science Publishers; 2006; Ch. 8: 249-71.

4. Stillwell W, Wassall SR. Docosahexaenoic acid: membrane properties of a unique fatty acid. Chem Phys Lipids 2003; 126L: 1-27.

5. Calder PC. Dietary modification of inflammation with lipids. Proc Nutr Soc 2002; 61: 345-58.

6. Giorgione J, Epand RM, Buda C, Farkas T. Role of phospholipids containing docosahexaenoyl chains in modulating the activity of protein kinase C. Proc Natl Acad Sci U S A 1995; 92: 9767-70.

7. Jump DB. Dietary polyunsaturated fatty acids and regulation of gene transcription. Curr Opin Lipidol 2002; 13: 155-64.

8. Kubo K, Sekine S, Saito M. Docosahexaenoic acidcontaining phosphatidylethanolamine in the external layer of liposomes protects docosahexaenoic acid from 2, 2'-azobis(2-aminopropane)dihydrochloride-mediated 
lipid peroxidation. Arch Biochem Biophys 2003; 410: 141-8.

9. Dratz EA, Van Breeman JF, Kamps KM, Keegstra W, Van Bruggen EF. Two-dimensional crystallization of bovine rhodopsin. Biochim Biophys Acta 1985; 832: $337-42$.

10. Feller SE, Gawrisch K, MacKerrell AD. Polyunsaturated fatty acids in lipid bilayers: intrinsic and environmental contributions to their unique physical properties. J Am Chem Soc 2002; 124: 318-26.

11. Shaikh SR, Brzustowicz MR, Stillwell W, Wassall SR. Formation of hexagonal phase in SDPE as observed by solid state ${ }^{31} \mathrm{P}$ NMR. Biochem Biophys Res Commun 2001; 286: 758-63.

12. Epand RM. Lipid polymorphism and protein-lipid interactions. Biochim Biophys Acta 1998; 1367: 353-68.

13. Miljanich GP, Sklar LA, White DL, Dratz EA. Disaturated and dipolyunsaturated phospholipids in the bovine retinal rod outer segment disk membrane. Biochim Biophys Acta 1979; 552: 294-306.

14. Anderson RE, Sperling L. Lipids of the ocular tissue VII. Positional distribution of the fatty acids in the phospholipids of bovine rod outer segments. Arch Biochem Biophys 1971; 144: 673-7.

15. Emmelot P, van Hoeven RP. Phospholipid unsaturation and plasma membrane organization. Chem Phys Lipids 1975; 14: 236-46.

16. Knapp H, Hullin F, Salem N Jr. Asymmetric incorporation of dietary n-3 fatty acids into membrane aminophospholipids of human erythrocytes. J Lipid Res 1994; 35: 1283-91.

17. Zerouga M, Stillwell W, Stone J, Powner A, Dumaual AC, Jenski LJ. Phospholipid class as a determinant in docosahexaenoic acid's effect on tumor cell viability. Anticancer Res 1996; 16: 2863-8.

18. Huster D, Jin AJ, Arnold K, Gawrisch K. Water permeability of polyunsaturated lipid membranes measured by ${ }^{17}$ O NMR. Biophys J 1997; 73: 855-64.

19. Wassall SR, Shaikh SR, Brzustowicz MR, Cherezov V, Siddiqui RA, Caffrey M, Stillwell W. Interaction of polyunsaturated fatty acids with cholesterol: a role in lipid raft phase separation. In: Danino D, Harries D, Wrenn SP, eds. Talking about colloids. Macromolecular Symposia. Vol 219. New York: Wiley-VCH; 1995. p. $73-84$.

20. Brown DA, London E. Structure and function of sphingolipid- and cholesterol-rich membrane rafts. J Biol Chem 2000; 275: 17221-4.

21. Edidin M. The state of lipid rafts: from model membranes to cells. Annu Rev Biophys Biomol Struct 2003; 32: $257-83$.

22. Racker E. A new look at mechanisms in bioenergetics. New York: Academic Press; 1976.

23. Shaikh SR, Dumaual AC, Castillo A, LoCascio D, Siddiqui RA, Stillwell W, Wassall SR. Oleic and docosahexaenoic acid differentially phase separate from lipid raft molecules: a comparative NMR, DSC, AFM and detergent extraction study. Biophys J 2004; 87: $1752-66$.

24. Niu S-L, Litman BJ. Determination of membrane cholesterol partition coefficient using a lipid vesiclecyclodextrin binary system: effect of phospholipid acyl chain unsaturation and head group composition. Biophys J 2002; 83: 3408-15.

25. Mitchell DC, Litman BJ. Modulation of receptor signaling by phospholipid acyl chain composition. In: Mostofsky D, Yehuda S, Salem N Jr, eds. Fatty acids: physiological and behavioral functions. Totowa, NJ: Humana Press; 2001. p. 23-40.

\section{William Stillwell}

Department of Biology Indiana University-Purdue University Indianapolis

723 West Michigan

Indianapolis, IN 46202 USA

Tel: + I 3172749580

E-mail: wstillwe@iupui.edu 